\title{
Algorithms for the Recognition of Net-free Graphs and for Computing Maximum Cardinality Matchings in Claw-free Graphs
}

\author{
Mihai Talmaciu', Victor Lepin ${ }^{2}$ \\ 1 "Vasile Alecsandri" University of Bacău, Romania, \\ mtalmaciu@ub.ro \\ 2 The Institute of Mathematics of the National Academy of Sciences of Belarus, \\ lepin@im.bas-net.by
}

\begin{abstract}
During the last decades, numerous studies have been undertaken on the classes of net-free graphs, claw-free graphs, and the relationship between them. The notion of weakly decomposition (a partition of the set of vertices in three classes $A, B, C$ such that $A$ induces a connected graph and $C$ is totally adjacent to $B$ and totally non-adjacent to $A$ ) and the study of its properties allow us to obtain several important results such as: characterization of cographs, $\left\{\mathrm{P}_{4}, \mathrm{C}_{4}\right\}$-free and paw-free graphs. In this article, we give a characterization of net-free graphs, a characterization of claw-free graphs, using weakly decomposition. Also, we give a recognition algorithm for net-free graphs, an algorithm for determining a maximum matching in claw-free graphs, comparable with existing algorithms in terms of complexity, but using weakly decomposition.
\end{abstract}

Keywords: net-free graphs, claw-free graphs, asteroidal triple-free graphs, weakly decomposition, recognition algorithm, maximum matching in graphs.

\section{Introduction}

A graph is claw-free if it has no induced subgraph isomorphic to the claw, i.e., the fourvertex star $K_{1,3}=\left(\left\{a_{1}, a_{2}, a_{3}, b\right\},\left\{b a_{1} \cdot b a_{2}, b a_{3}\right\}\right)$. A net is a graph obtained from a triangle by attaching to each vertex a new dangling edge.

The interval graphs [24], permutation graphs [16] and co-comparability graphs [18] have a linear structure. Each of these classes is a subfamily of the asteroidal triple graphs (ATfree graphs, for short). An independent set of three vertices is called an asteroidal triple if between any pair in the triple there exists a path that avoids the neighbourhood of the third. ATfree graphs were introduced by Lekkerkerker and Boland [24]. Corneil, Olariu and Stewart showed a number of results on the linear structure of AT-free $[8,9,10]$.

A maximal subclass of a class of net-free graphs is the class (claw,net)-free graphs $(\mathrm{CN}$ free graphs, for short). Also note that $\mathrm{CN}$-free graphs are exactly the Hamiltonian-hereditary graphs[13] (was cited in [4]). CN-free graphs turn out to be closely related to AT-free graphs form their structure properties [4]. There are, however, few results about the structure of these graphs [4]. In [4] the authors give results on the linear and circular structure of $\mathrm{CN}$-free graphs. AT-free graphs can be generalized in a manner obvious to admit circular structure [4].
$\mathrm{CN}$-free graphs were introduced by Duffus [14]. Although $\mathrm{CN}$-free graphs seems to be quite restrictive, it contains a couple of families of graphs that are interesting in their own right.

In this paper we give an algorithm for the recognition of net-free graph of complexity $\mathrm{O}\left(\mathrm{n}\left(\mathrm{n}+\mathrm{m}^{1,63}\right)\right)$. Also, we give an interesting property of claw-free graph that leads to a algorithm for the construction a maximum matching in claw-free graphs.

The content of the paper is organized as follows. In Preliminaries, we give the usual terminology in graph theory. In Section 3 we give a characterization of net-free graphs and a recognition algorithm using the weakly decomposition. In Section 4 we determine a maximum matching in the claw-free graph. Ideas for future work conclude the paper.

\section{Preliminaries}

Throughout this paper, $G=(V, E)$ is a connected, finite and undirected graph ([3]), without loops and multiple edges, having $V=V(G)$ as the vertex set and $E=E(G)$ as the set of edges. $\bar{G}$ is the complement of $G$. If $U \subseteq V$, by $G(U)$ we denote the subgraph of $G$ induced by $U$. By $G$ $X$ we mean the subgraph $G(V-X)$, whenever $X \subseteq V$, but we simply write $G$ - $v$, when $X=\{v\}$. If $e=x y$ is an edge of a graph $\mathrm{G}$, then $x$ and $y$ are adjacent, while $x$ and $e$ are incident, as are $y$ 
and $e$. If $x y \in E$, we also use $x \sim y$, and $x \wedge y$ whenever $x, y$ are not adjacent in $G$. If $A, B \subseteq V$ are disjoint and $a b \in E$ for every $a \in A$ and $b \in B$, we say that A,B are totally adjacent and we denote by $A \sim B$, while by $A \backsim B$ we mean that no edge of $G$ joins some vertex of $A$ to a vertex from $B$ and, in this case, we say $A$ and $B$ are totally non-adjacent.

The neighborhood of the vertex $v \in V$ is the set $N_{G}(v)=\{u \in V: u v \in E\}, \quad$ while $N_{G}[v]=N_{G}(v) \cup\{v\}$; we denote $N(v)$ and $N[v]$, when $G$ appears clearly from the context. The degree of $v$ in $G$ is $d_{G}(v)=\left|N_{G}(v)\right|$. The neighborhood of the vertex $v$ in the complement of $G$ will be denoted by $\bar{N}(v)$.

The neighborhood of $S \subseteq V$ is the set $N(S)=\cup_{v \in S} N(v)-S$ and $N[S]=S \cup N(S)$. A graph is complete if every pair of distinct vertices is adjacent.

By $P_{n}, C_{n}, K_{n}$ we mean a chordless path on $n \geq 3$ vertices, a chordless cycle on $n \geq 3$ vertices, and a complete graph on $n \geq 1$ vertices, respectively.

Let $F$ denote a family of graphs. A graph $G$ is called $F$-free if none of its subgraphs are in $F$.

The Zykov sum of the graphs $G_{1}, G_{2}$ is the graph $G=G_{1}+G_{2}$ having:

$V(G)=V\left(G_{1}\right) \cup V\left(G_{2}\right)$,

$\mathrm{E}(\mathrm{G}) \quad=\mathrm{E}\left(\mathrm{G}_{1}\right) \cup \mathrm{E}\left(\mathrm{G}_{2}\right) \cup\left\{\mathrm{uv}: \mathrm{u} \in \mathrm{V}\left(\mathrm{G}_{1}\right), \mathrm{v} \in\right.$ $\left.\mathrm{V}\left(\mathrm{G}_{2}\right)\right\}$.

\section{A New Characterization of Net-free Graphs using the Weakly Decomposition}

Formation control is an important issue of motion coordination of Multi-agent Robots Systems, it is based on the properties of the formation graphs ([20]).

We recall a characterization of the weakly decomposition of a graph.

Definition 1. ([32], [33]) A set $A \subseteq V(G)$ is called a weakly set of the graph $G$ if $N_{G}(A) \neq V(G)$-A and $G(A)$ is connected. If $A$ is a weakly set, maximal with respect to set inclusion, then $G(A)$ is called a weakly component. For simplicity, the weakly component $G(A)$ will be denoted with $A$.
Definition 2. ([32], [33]) Let $G=(V, E)$ be a connected and non-complete graph. If $A$ is a weakly set, then the partition $\{A, N(A), V$ $A \cup N(A)\}$ is called a weakly decomposition of $G$ with respect toA.

The name of weakly component is justified by the following result.

Theorem 1.([32], [33]) Every connected and non-complete graph $G=(V, E)$ admits a weakly component $A$ such that $G(V$ $A)=G(N(A))+G(\bar{N}(A))$.

Theorem 2.([11], [12]) Let $\mathrm{G}=(\mathrm{V}, \mathrm{E})$ be a connected and non-complete graph and $\mathrm{A} \subseteq \mathrm{V}$. Then $\mathrm{A}$ is a weakly component of $G$ if and only if $G(A)$ is connected and $N(A) \sim \bar{N}(A)$.

The next result, that follows from Theorem 2, ensures the existence of a weakly decomposition in a connected and noncomplete graph.

Corollary 1. If $G=(V, E)$ is a connected and non-complete graph, then $V$ admits a weakly decomposition $(A, B, C)$, such that $G(A)$ is a weakly component and $G(V-A)=G(B)+G(C)$.

Theorem 2 provides an $O(n+m)$ algorithm for building a weakly decomposition for a noncomplete and connected graph.

Algorithm for the weakly decomposition of a graph ([32])

Input: A connected graph with at least two nonadjacent vertices, $G=(V, E)$.

Output: A partition $\mathrm{V}=(\mathrm{A}, \mathrm{N}, \mathrm{R})$ such that $\mathrm{G}(\mathrm{A})$ is connected, $\mathrm{N}=\mathrm{N}(\mathrm{A}), \mathrm{A} \leftrightarrow \mathrm{R}=\bar{N}(\mathrm{~A})$.

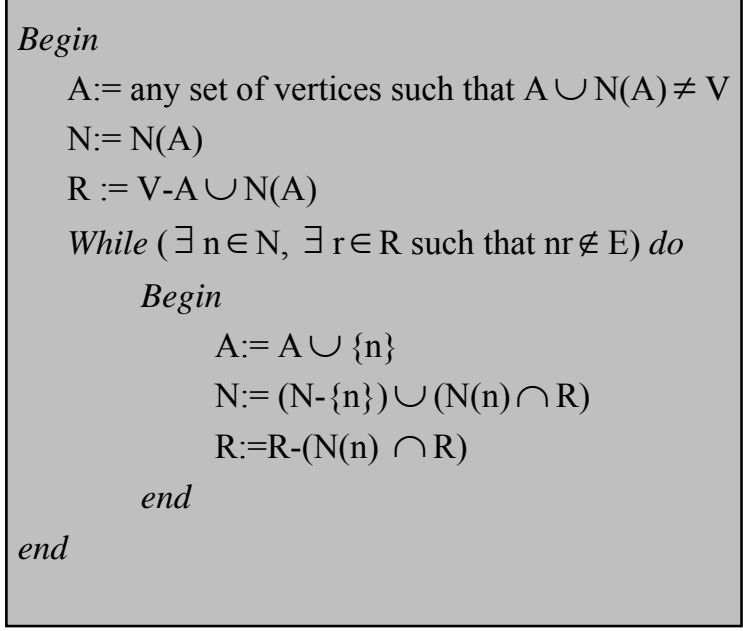

A new characterization of net-free graphs, using weakly decomposition, is given below. 
Theorem 3. Let $G=(V, E)$ be a connected and non-complete graph. Let $(\mathrm{A}, \mathrm{N}, \mathrm{R})$ be a weakly decomposition with $\mathrm{G}(\mathrm{A})$ a weakly component. $\mathrm{G}=(\mathrm{V}, \mathrm{E})$ is net-free if and only if:

i) does not exist $\mathrm{P}_{4}$ in $\mathrm{G}(\mathrm{A})$ and $\mathrm{n}$ in $\mathrm{N}$ such that $\mathrm{n}$ is adjacent with the middle vertices of the $\mathrm{P}_{4}$ specified;

ii) (does not exist $\mathrm{P}_{4}$, with extremities in $\mathrm{A}$ and the middle vertices in $\mathrm{N}$ ) or (does not exist $t$ in $\mathrm{N}$ such that his neighbours $\mathrm{t}$ are not in $\mathrm{P}_{4}$ specified);

iii) $\mathrm{G}(\mathrm{V}-\mathrm{R}), \mathrm{G}(\mathrm{V}-\mathrm{A})$ are net-free.

Proof.\} Let $\mathrm{G}$ be net-free. Since the property of being net-free is hereditary as follows $\mathrm{G}(\mathrm{V}-\mathrm{A})$ and $\mathrm{G}(\mathrm{V}-\mathrm{R})$ net-free graphs, so iii) holds. If $\exists \mathrm{n} \in \mathrm{N}, \exists \mathrm{P}_{4} \subseteq \mathrm{G}(\mathrm{A})$ such that $\mathrm{n}$ is adjacent to the middle vertices in $\mathrm{P}_{4}$, so $\exists \mathrm{P}_{4}$ : a, b, c, d with $a, b, c, d \in A, a b, b c, c d \in E, a c, a d, b d \notin E$ and $n b, n c \in E$, then, because $N \sim R$, it follows that $\forall \mathrm{r} \in \mathrm{R}: \mathrm{G}(\{\mathrm{a}, \mathrm{b}, \mathrm{c}, \mathrm{d}, \mathrm{n}, \mathrm{r}\})$ is net, a contradiction. So i) holds. If $\exists \mathrm{P}_{4}$ : ab, bc, cd with $\mathrm{a}, \mathrm{d} \in \mathrm{A}, \mathrm{b}, \mathrm{c} \in \mathrm{N}$ and $\exists \mathrm{t} \in \mathrm{N}$ with ta,tb, tc, td $\notin \mathrm{E}$ then $\mathrm{G}(\{\mathrm{a}, \mathrm{b}, \mathrm{c}, \mathrm{d}, \mathrm{r}, \mathrm{t}\})$ is net $\forall \mathrm{r} \in \mathrm{R}$, a contradiction. So ii) holds.

Conversely, we suppose that i), ii), iii) hold and to show that $\mathrm{G}$ is net-free. From iii) it follows that $G(A), G(N), G(R), G(A \cup N)$ and $G(N$ $\cup R)$ are net-free. Because $G(A \cup R)$ is not connected it follows that $G(A \cup R)$ is net-free. Suppose, however, that there is $\mathrm{H}=\mathrm{G}(\{\mathrm{a}, \mathrm{b}, \mathrm{c}, 1,2,3\})$ an subgraph net, with the vertices $a, b, c$ of the degree 1 , the vertices 1,2 , 3 of degree 3 , and a $1, \mathrm{~b} 2$, c $3 \in \mathrm{E}$.

Case 1. Let $|\mathrm{V}(\mathrm{H}) \cap \mathrm{R}|=1$. We assume 1.1. $\mathrm{V}(\mathrm{H}) \cap \mathrm{R}=\{\mathrm{a}\}$. 1.2. $\mathrm{V}(\mathrm{H}) \cap \mathrm{R}=\{1\} .1 .1$. From $\mathrm{R} \sim \mathrm{N}$ it follows $\mathrm{V}(\mathrm{H}) \cap \mathrm{N}=\{1\}$. So $\mathrm{V}(\mathrm{H})$ $\cap A=\{c, 3,2, b\}$. But $G(\{c, 3,2, b\})$ is $P_{4}$ and 1 is adjacent with the middle vertices in $\mathrm{P}_{4}$, thereby contradicting with i). 1.2. From $\mathrm{R} \sim \mathrm{N}$ it follows $\mathrm{V}(\mathrm{H}) \cap \mathrm{N}=\{\mathrm{a}, 2,3\}$. So $\mathrm{V}(\mathrm{H}) \cap \mathrm{A}=\{\mathrm{b}, \mathrm{c}\}$. $\mathrm{P}=\mathrm{G}(\{\mathrm{b}, \mathrm{c}, 2,3\})$ is an $\mathrm{P}_{4}$, with extremities $\mathrm{b}$, c $\in A$ and the middle vertices $2,3 \in \mathrm{N}$. For $\mathrm{t}=\mathrm{a}$ we have $\mathrm{N}(\mathrm{t}) \cap \mathrm{V}(\mathrm{P})=\phi$, thereby contradicting with ii). So, Case 1 holds not.

Case 2. Let $|\mathrm{V}(\mathrm{H}) \cap \mathrm{R}|=2$. There are subcases 2.1. $\mathrm{V}(\mathrm{H}) \cap \mathrm{R}=\{\mathrm{a}, 1\} ; 2.2$. $\mathrm{V}(\mathrm{H}) \cap \mathrm{R}=\{1,2\}$; 2.3. $\mathrm{V}(\mathrm{H}) \cap \mathrm{R}=\{\mathrm{a}, 2\} ; 2.4 . \mathrm{V}(\mathrm{H}) \cap \mathrm{R}=\{\mathrm{a}, \mathrm{b}\}$.

2.1.cannot hold because the vertices a and 1 have no common neighbors. 2.2. cannot hold because the vertices 1 and 2 not have only common neighbors. 2.3. cannot hold because the vertices a and 2 not have only common neighbors. 2.4. cannot hold because the vertices $\mathrm{a}$ and $\mathrm{b}$ have no common neighbours.

Case 3. $|\mathrm{V}(\mathrm{H}) \cap \mathrm{R}|=3$ cannot hold because the vertices in $\{a, 1,2\}$ and in $\{a, 1, b\}$ not have only common neighbours.

Case 4. Let $|\mathrm{V}(\mathrm{H}) \cap \mathrm{R}|=4$. Any subset $\mathrm{X} \subseteq \mathrm{V}(\mathrm{H})$ with $|\mathrm{X}|=4$ has the property that its vertices are not only common neighbours, that is $\exists \mathrm{v} \in \mathrm{V}$-X such that $\mathrm{v}$ is adjacent some of the vertices of $X$, and with the rest of them, $v$ it is not adjacent.

$|\mathrm{V}(\mathrm{H}) \cap \mathrm{R}| \in\{5,6\}$ it is not possible, because $\mathrm{V}(\mathrm{H}) \cap \mathrm{A} \neq \phi$ si $\mathrm{V}(\mathrm{H}) \cap \mathrm{N} \neq \phi$.

Theorem 3 provides the following recognition algorithm for net-free graphs.

Algorithm Recognition

Input: A connected, non-complete graph $\mathrm{G}=(\mathrm{V}, \mathrm{E})$.

Output: An answer to the question: "Is G net-free"?

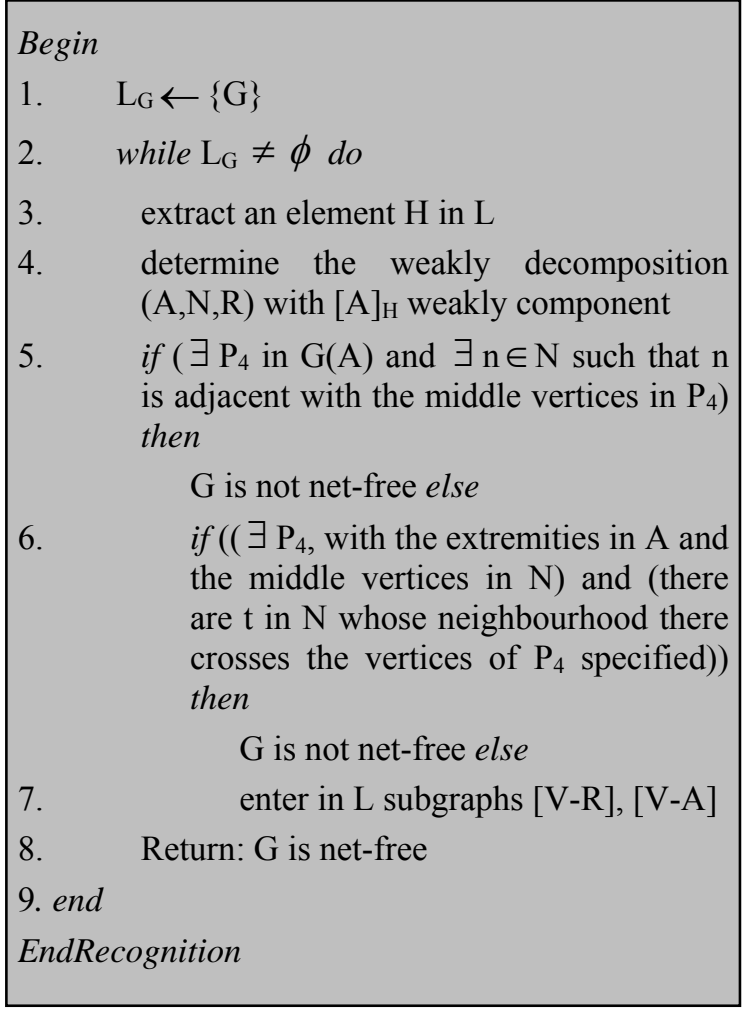

As Theorem 2 provides an $O(n+m)$ algorithm for building a weakly decomposition for a noncomplete and connected graph and as for the recognition $\mathrm{P}_{4}$-free in $O(n+m)$ ([17]) time (Finding small cycles in undirected graphs in $\mathrm{O}\left(\mathrm{m}^{1,63}\right)([1])$ time), so step 5 and step 6 run in $O\left(n+\mathrm{m}^{1,63}\right)$ time, it follows that, in total, the algorithm is run in $O\left(n\left(n+\mathrm{m}^{1,63}\right)\right)$. 


\section{Determining a Maximum Matching within a Claw-free Graph}

Software testing is an important process that helps to develop high quality software. Test data generation can be done using combinatorial optimizing techniques. ([28]).

Some interesting properties of claw-free graphs have been established in ([2], [6], [17], [22], [25], [30]). In [21] the authors present new algorithms for the recognition of claw-free graphs $\left(O\left(e^{1,69}\right)\right)$. In [7] the authors mention the structure theory of claw-free graphs.

In [5] the authors consider the algorithmic problem of finding a Hamiltonian path or a Hamiltonian cycle efficiently.

In what follows we characterize the claw-free graphs and we give an algorithm for constructing a cardinal maximum matching in a claw-free graph.

A similar result is found in ([32])

Theorem 4. Let $G=(V, E)$ be a connected and non-complete graph. Let $(A, N, R)$ a weakly decomposition with $G(A)$ a weakly component. $G=(V, E)$ is claw-free if and only if:

i) $R$ and $N(n) \cap A$ are cliques, $\forall n \in N$

ii) $G(V-R), G(V-A)$ are claw-free.

Proof.\} Let $\mathrm{G}$ be a claw-free graph. From he heredity of the claw-free graphs it follows that $\mathrm{G}(\mathrm{V}-\mathrm{A})$ and $\mathrm{G}(\mathrm{V}-\mathrm{R})$ are claw-free graphs. If there would be $r_{1}, r_{2} \in R$ such that $r_{1} r_{2} \notin E$, because $\mathrm{R} \sim \mathrm{N}, \forall \mathrm{n} \in \mathrm{N}$ and $\mathrm{a} \in \mathrm{N}(\mathrm{n}) \cap \mathrm{A}$ (N(n) $\cap \mathrm{A} \neq \phi, \forall \mathrm{n} \in \mathrm{N}$ according to his $\mathrm{N}$ ), $\mathrm{G}\left(\left\{\mathrm{a}, \mathrm{n}, \mathrm{r} \_1, \mathrm{r} \_2\right\}\right)$ is isomorphic to claw.

Show of the reverse implication. We assume that there are $\{\mathrm{x}, \mathrm{a}, \mathrm{b}, \mathrm{c}\}$ a claw with center in $\mathrm{x}$. From ii) results that $G(A \cup N)$ and $G(N \cup R)$ are claw-free graphs. So $x \notin A \cup R$, that is $x \in N$. From i), two of the vertices $a, b, c$ are necessarily in $\mathrm{N}$, that is $\{\mathrm{x}, \mathrm{a}, \mathrm{b}, \mathrm{c}\}$ are in $\mathrm{A} \cup \mathrm{N}$ or in $N \cup R$, thereby contradicting with ii).

Theorem 4 leads to a recognition algorithm for a claw-free graph that shows the combinatorial structure of the graph the decomposition mode used. Because the complexity, of this algorithm is not better than the most efficient known, not present.

Sumner ([31]) and, independently, Las Vergnas ([23]) proved that every claw-free connected graph with an even number of vertices has a perfect matching.

It is known that if $\mathrm{G}$ is a connected graph there is a vertex $v \in V(G)$ such that $G-v$ is connected.

In [29], Sumner shows that in any connected claw-free graphs one can find a pair of adjacent vertices the removal of which leaves the remaining graphs connected.

An interesting question would be to determine the connected graphs with the $\exists \mathrm{Q} \subset \mathrm{V}(\mathrm{G})$, Q the maximum clique in relation to inclusion so that G-Q is connected. This property is related to the existence of induced subgraphs claw. The following result.

Theorem 5. Let $G=(V, E)$ be a connected and non-complete graph. Let $(A, N, R)$ be a weakly decomposition with $G(A)$ a weakly component. $G$ is claw-free if and only if for any induced subgraph $H$ of $G, H$ connected, there is a maximal clique $Q$ in $H$ such that $H$ $Q$ is connected.

Proof. Let G be a connected and claw-free graph. Because the property of being claw-free is hereditary, $\forall \mathrm{H}$, induced subgraph of $\mathrm{G}, \mathrm{H}$ connected, $\mathrm{H}$ is claw-free. From Theorem 7 it follows that $\mathrm{R}$ is clique. Let $\mathrm{Q} \subseteq \mathrm{N}$ be a maximal clique in $[\mathrm{N}]_{\mathrm{H}}$. Then $\mathrm{Q}_{1}=\mathrm{Q} \cup \mathrm{R}$ is a maximal clique in $\mathrm{H}$ (in $\mathrm{N} \sim \mathrm{R}$ it follows that $\mathrm{Q}_{1}$ is clique; $\mathrm{Q}_{1}$ is maximal because $\forall \mathrm{x} \in \mathrm{V}(\mathrm{H})-\mathrm{Q}_{1}$, we have or $\mathrm{x} \in \mathrm{A}$ and $\mathrm{xr} \notin \mathrm{E}(\mathrm{H}), \forall \mathrm{r} \in \mathrm{Q}_{1} \cap \mathrm{R}$ or $\mathrm{x} \in \mathrm{N}-\mathrm{Q}$ and because $\mathrm{Q}$ is maximal, $\exists \mathrm{y} \in \mathrm{Q}_{1} \cap \mathrm{N}$ such that $\mathrm{x} \mathrm{y} \notin \mathrm{E}(\mathrm{H}))$. Because $[\mathrm{A}]_{\mathrm{H}}$ is connected and $\forall \mathrm{x} \in \mathrm{N}-\mathrm{Q}_{1}, \quad \exists \mathrm{a} \in \mathrm{A}$ such that $\mathrm{ax} \in \mathrm{E}(\mathrm{H})$, it follows that $\mathrm{H}-\mathrm{Q}_{1}$ is connected.

Show of the reverse implication. We assume that there is $\mathrm{H}$ isomorphic a induced claw in $\mathrm{G}$, $H=G(\{x, a, b, c\}), x$ being the center of $H$. Then the maximum cliques of $H$ are $\{x, a\},\{x, b\}$, $\{\mathrm{x}, \mathrm{c}\}$ and every one of them disconnects $\mathrm{H}$.

As Edmonds ([15]) showed, a maximum matching in any graph may be found in polynomial time. Sbihi ([27]) extended this algorithm to one that computes a maximum independent set in any claw-free graph.Minty ([26]) (corrected by Nakamura, Tamura [27]) independently provided an alternative extension of Edmonds' algorithms to claw-free graphs.

Theorem 5 provides an algorithm for constructing a cardinal maximum matching in a claw-free graph.

Input: A connected, non-complete, claw-free graph 
$\mathrm{G}=(\mathrm{V}, \mathrm{E})$.

Output: A cardinal maximum matching in $\mathrm{G}$

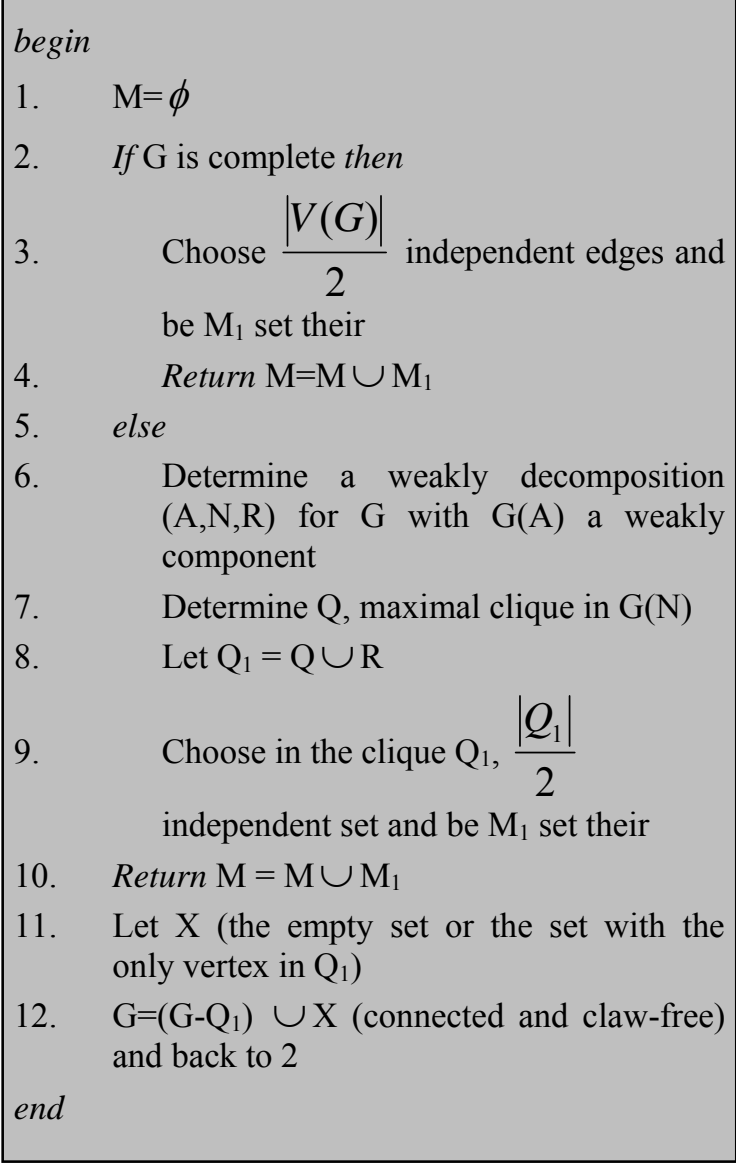

Step 7 is run in $O(n(n+m))\}$

\begin{tabular}{|ll}
\hline Begin & \\
71. & $\mathrm{~L}_{\mathrm{G}} \leftarrow\{\mathrm{G}(\mathrm{N})\}$ \\
72. & $\mathrm{Q}=\phi$ \\
73. & while $\mathrm{L}_{\mathrm{G}} \neq \phi$ do \\
74. & extract an element $\mathrm{H}$ in $\mathrm{L}_{\mathrm{G}}$ \\
75. & determine the weakly decomposition \\
& $(\mathrm{A}, \mathrm{N}, \mathrm{R}) \quad$ with $\quad[\mathrm{A}]_{\mathrm{H}} \quad$ weakly \\
& component \\
76. & because $\mathrm{G}(\mathrm{N})$ is claw-free, $\mathrm{R}$ clique \\
& and $\mathrm{R} \sim \mathrm{N}$ \\
77. & $\mathrm{Q} \leftarrow \mathrm{Q} \cup \mathrm{R}$ \\
End & end $\}$ \\
&
\end{tabular}

As Theorem 2 provides an $O(n+m)$ algorithm for building a weakly decomposition for a noncomplete and connected graph, in total, the algorithm is run in $O(n(n(n+m)))$.

\section{Conclusions and Future Work}

In this paper we have given a recognition algorithm for net-free graphs and an algorithm for determining a maximum matching in clawfree graphs. Our future work is going to put forward some applications of \{net,claw\}-free.

\section{Acknowledgment}

The current research of Mihai Talmaciu was supported by the project entitled Classes of graphs, complexity of problems and algorithms, "AR-FRBCF", 2012-2013, a Bilateral Cooperation Research Project, involving Romanian Academy, National Academy of Sciences of Belarus, Republican Foundation for Fundamental Research and "Vasile Alecsandri" University of Bacau.

\section{REFERENCES}

1. ALON, N., R. YUSTER, U. ZWICK, Finding and Counting Given Length Cycles, Proc. 2nd European Symposium on Algorithms, Utrecht, The Netherlands, 1994, pp. 354-364

2. ASRATIAN, A. S., Every 3-connected, Locally Connected, Claw-free Graph is Hamilton-connected, J. Graph Theory, vol. 23, 1996, pp. 191-201.

3. BERGE, C. Graphs, Amsterdam, 1985.

4. BRANDSTEDTA, A., F. F. DRAGAN, On Linear and Circular Structure of (Claw, Net)-free Graphs, Discrete Applied Mathematics, vol. 129(23), 2003, pp. 285-303.

5. BRANDSTADT, A., F. F. DRAGAN, E. KOHLER, Linear Time Algorithms for Hamiltonian Problems on (Claw, Net)free Graphs, SIAM J. COMPUT., Vol. 30(5), 2000, pp. 1662-1677.

6. BROUSEK, J., Minimal 2-connected Non-Hamiltonian Claw-free Graphs, Discrete Math, vol. 191, 1998, pp. 57-64.

7. CHUDNOVSKY, M., P. SEYMOUR, The Structure of Claw-free Graphs, Surveys in Combinatorics, London Math. Soc. Lecture Note Ser. 327, Cambridge: Cambridge Univ. Press, 2005, pp. 153-171.

8. CORNEIL, D. G., S. OLARIU, L. STEWART, A Linear Time Algorithm to Compute a Dominating Path in an AT-free Graph, Inform. Proc. Lett. Vol. 54, 1995, pp. 253-257.

9. CORNEIL, D. G., S. OLARIU, L. STEWART, Asteroidal Triple-free Graphs, SIAM J. Discrete Math., vol. 10, 1997, pp. 399-430. 
10. CORNEIL, D. G., S. OLARIU, L. STEWART, Linear Time Algorithms for Dominating Pairs in Asteroidal Triple-free Graphs, SIAM J. Comput. vol. 28, 1999, pp. 1284-1297.

11. CROITORU, C., E. OLARU, M. TALMACIU, Confidentially connected Graphs, The annals of "Dunarea de Jos" Univ. of Galati, Proc. of the Intl. Conf. "The Risk in Contemporary Economy", Suppl. to Tome XVIII (XXII), 2000, pp. 17-20.

12. CROITORU, C., M. TALMACIU, On Confidentially Connected Graphs, Bul. Stiint. Univ. Baia Mare, Ser B, Matematica - Informatica, vol. XVI(1), 2000, pp. 13-16.

13. DAMASCHKE, P., Hamiltonianhereditary Graphs, unpublished.

14. DUFFUS, D., M. S. JACOBSON, R. J. GOULD, Forbidden Subgraphs and the Hamiltonian Theme, in The Theory and Applications of Graphs, (Kalamazoo, Mich, 1980), Wiley, New York, pp. 297-316.

15. EDMONDS J., Paths, Trees and Flowers, Canadian Journal of Math, vol. 17, 1965, pp. 449-467.

16. EVEN, S., A. PNUELI, A. LEMPEL, Permutation Graphs and Transitive Graphs, J. ACM, vol. 19, 1972, pp. 400-410.

17. FLANDRIN, E., J. L. FOUQUET, H. LI, On Hamiltonian Claw-free Graphs, Discrete Math., vol. 111, 1993, pp. 221-229.

18. GOLUMBIC, M. C., C. L. MONMA, W. T. TROTTER Jr., Tolerance Graphs, Discrete Applied Mathematics, vol. 9, 1984, pp. 157-170.

19. HABIB, M., C. PAUL, A Simple Linear Time Algorithm for Cograph Recognition, Discrete Applied Mathematics, vol. 145, 2005, pp. 183-197.

20. HERNANDEZ-MARTINEZ, E. G., E. ARANDA-BRICAIRE, Decentralized Formation Control of Multi-agent Robot Systems based on Formation Graphs, Studies in Informatics and Control, vol. 21(1), pp. 7-16, 2012.

21. KLOKS, T., D. KRATSCH, H. MÜLLER, Finding and Counting Small Induced Subgraphs Efficiently, Information Proc. Letters, vol. 74(34), 2000, pp. 115-121
22. KOHLER, E., Linear Time Algorithms for Hamiltonian Problems in Claw-Free AT-Free Graphs, manuscript, 1999.

23. Las VERGNAS, M. A Note on Matchings in Graphs, Cahiers du Centre d'etudes de Recherche Operationnelle, vol. 17(2-3-4), 1975, pp. 257-260.

24. LEKKERKERKER, C. G., J. C. BOLAND, Representation of a Finite Graph by a Set of Intervals on the Real Line, Fund. Math., vol. 51, 1962, pp. 45-64.

25. LI, M., Hamiltonian Cycles in 3Connected Claw-free Graphs, J. Graph Theory, vol. 17, 1993, pp. 303-313.

26. MINTY, G. J., On Maximal Independent Sets of Vertices in Claw-free Graphs, Journal of Combinatorial Theory. Series B, vol. 28(3), 1980, pp. 284-304.

27. NAKAMURA, D., A. TAMURA, A Revision of Minty's Algorithm for Finding a Maximum Weighted Stable Set of a Claw-free Graph, Journal of the Operations Research Society of Japan, vol. 44(2), 2001, pp. 194-204.

28. POCATILU, P., I. IVAN, A Genetic Algorithm-based System for Automatic Control of Test Data Generation, Studies in Informatics and Control, vol. 22(2), 2013, pp. 219-226.

29. SBIHI, N., Algorithme de recherche d'un stable de cardinalite maximum dans un graphe sans etoile, Discrete Mathematics, vol. 29(1), 1980, pp. 53-76.

30. SHEPHERD, F. B., Hamiltonicity in Claw-free Graphs, J. Combin. Theory Ser. B, vol. 53, 1991, pp. 173-194.

31. SUMNER, D. P., Graphs with 1-factors, Proceedings of the American Mathematical Society (American Mathematical Society), vol. 42(1), 1974, pp. 8-12.

32. TALMACIU, M., Decomposition Problems in the Graph Theory with Applications in Combinatorial Optimization - Ph. D. Thesis, "Al. I. Cuza" University Iasi, Romania, 2002.

33. TALMACIU, M., E. NECHITA, Recognition Algorithm for Diamond-free Graphs, Informatica, vol.18(3), 2007, pp. 457-462. 